\title{
Onsager reciprocal relations with broken time-reversal symmetry
}

\author{
Rongxiang Luo $\odot,{ }^{1}$ Giuliano Benenti $\odot,{ }^{2,3,4}$ Giulio Casati, ${ }^{2,5}$ and Jiao Wang $\oplus^{1}$ \\ ${ }^{1}$ Department of Physics, Key Laboratory of Low Dimensional Condensed Matter Physics (Department of Education of Fujian Province), \\ and Jiujiang Research Institute, Xiamen University, Xiamen 361005, Fujian, China \\ ${ }^{2}$ Center for Nonlinear and Complex Systems, Dipartimento di Scienza e Alta Tecnologia, \\ Università degli Studi dell'Insubria, via Valleggio 11, 22100 Como, Italy \\ ${ }^{3}$ Istituto Nazionale di Fisica Nucleare, Sezione di Milano, via Celoria 16, 20133 Milano, Italy \\ ${ }^{4}$ NEST, Istituto Nanoscienze-CNR, I-56126 Pisa, Italy \\ ${ }^{5}$ International Institute of Physics, Federal University of Rio Grande do Norte, Campus Universitário-Lagoa Nova, Caixa Postale 1613, \\ Natal, Rio Grande Do Norte 59078-970, Brazil
}

(Received 20 August 2019; accepted 18 March 2020; published 10 April 2020)

\begin{abstract}
We provide analytical and numerical evidence that Onsager reciprocal relations remain valid for systems with broken time-reversal symmetry as is typically the case when a generic magnetic field is present. Our results show that the Onsager reciprocal relations are much more general than previously assumed. Hence, the fundamental constraints they impose on heat to work conversion remain valid also with broken time-reversal symmetry. In particular, the possibility of an engine operating at the Carnot efficiency with finite power is ruled out on purely thermodynamic grounds.
\end{abstract}

DOI: 10.1103/PhysRevResearch.2.022009

Introduction. Onsager reciprocal relations [1-3] are a cornerstone in nonequilibrium statistical physics. In the linear response regime, given a system brought out of equilibrium by the thermodynamic forces $\mathcal{F}_{k}$, the corresponding fluxes $J_{k}$ are such that, in the coupled transport equations $J_{j}=\sum_{k} L_{j k} \mathcal{F}_{k}$, the kinetic coefficients $L_{j k}$ obey the relations $L_{j k}=L_{k j}$. Onsager reciprocal relations reflect at the macroscopic level the time-reversal symmetry of the microscopic dynamics, which is invariant under the transformation $\mathcal{T}(\boldsymbol{r}, \boldsymbol{p}, t) \equiv(\boldsymbol{r},-\boldsymbol{p},-t)$, where $\boldsymbol{r}, \boldsymbol{p}$, and $t$ are coordinates, momenta, and time. Therefore, it is expected that Onsager relations are no longer valid when time-reversal symmetry is broken, typically by an applied magnetic field. In this case, the invariance of equations of motion is recovered if the time $t$ is replaced by $-t$ and simultaneously the magnetic field $\boldsymbol{B}$ by $-\boldsymbol{B}: \mathcal{T}_{\boldsymbol{B}}(\boldsymbol{r}, \boldsymbol{p}, t, \boldsymbol{B}) \equiv$ $(\boldsymbol{r},-\boldsymbol{p},-t,-\boldsymbol{B})$. This leads to the Onsager-Casimir relations $[1,4]$ for the kinetic coefficients: $L_{j k}(\boldsymbol{B})=L_{k j}(-\boldsymbol{B})$, while in principle one could still have $L_{j k}(\boldsymbol{B}) \neq L_{k j}(\boldsymbol{B})$, thus violating the Onsager symmetry.

As the principles of thermodynamics, Onsager relations introduce fundamental constraints on heat to work conversion. Violation of the Onsager symmetry, as expected when timereversal symmetry is broken, would allow one, in principle, to have finite power at Carnot efficiency [5]. Indeed, in such situation, the second law of thermodynamics is consistent with the possibility to have nondissipative currents, generated by thermodynamic forces.

Published by the American Physical Society under the terms of the Creative Commons Attribution 4.0 International license. Further distribution of this work must maintain attribution to the author(s) and the published article's title, journal citation, and DOI.
For concreteness, we consider the example of coupled charge and heat transport [6], where $\mathcal{F}_{e}$ and $\mathcal{F}_{h}$ are the electrochemical and temperature gradient, $J_{e}$ and $J_{h}$ the charge and heat flow, and the Onsager symmetry implies $L_{h e}=L_{e h}$. On the other hand, when time-reversal symmetry is broken by an applied magnetic field, the Onsager-Casimir relation $\Pi(\boldsymbol{B})=$ $T S(-\boldsymbol{B})$ holds $\left(\Pi=L_{h e} / L_{e e}\right.$ is the Peltier coefficient, $S=$ $L_{e h} / T L_{e e}$ the thermopower, and $T$ the temperature), while in principle one could have $\Pi(\boldsymbol{B}) \neq T S(\boldsymbol{B})$ [i.e., $L_{e h}(\boldsymbol{B}) \neq$ $\left.L_{h e}(\boldsymbol{B})\right]$, thus breaking the Onsager symmetry.

For $L_{e h} \neq L_{h e}$, the laws of thermodynamics would not forbid the existence of an engine working at the Carnot efficiency $\eta_{C}$ while delivering finite power [6] $P=\frac{\eta_{C}}{4} \frac{\left|L_{e h}^{2}-L_{h e}^{2}\right|}{L_{e e}} \mathcal{F}_{h}$. Such a spectacular possibility was denied in models with inelastic scattering [7-14] and for systems described as Markov processes [15]. On the other hand, a general no-go theorem based on purely thermodynamic considerations is not available.

There are special cases for which it is known that Onsager reciprocal relations remain valid in spite of an applied magnetic field: for noninteracting systems connected to two reservoirs, as a consequence of the symmetry properties of the scattering matrix [16], and for interacting systems subject to a constant magnetic field [17-20]. Then the relevant question arises: is it possible that Onsager relations remain valid under general broken time-reversal symmetry as in the case of a generic spatially dependent magnetic field? In this Rapid Communication, we shall show, on the basis of analytical results and extensive numerical simulations, that this is the case. Our results show that the Onsager reciprocal relations are much more general than previously assumed. Consequently, breaking the time-reversal symmetry does not remove the thermodynamic constraints imposed by the Onsager relations, and the above possibility of an engine with 
nonvanishing power at the Carnot efficiency is ruled out on purely thermodynamic grounds.

Analytical result. We consider a system of $N$ interacting particles, governed by the Hamiltonian

$$
H=\sum_{i}^{N} \frac{\left[\boldsymbol{p}_{i}-q_{i} \boldsymbol{A}\left(\boldsymbol{r}_{i}\right)\right]^{2}}{2 m_{i}}+\frac{1}{2} \sum_{i} \sum_{j \neq i} V\left(r_{i j}\right),
$$

where $\boldsymbol{r}_{i}$ and $\boldsymbol{p}_{i}$ are the conjugated coordinates and momenta of particle $i$ (of mass $m_{i}$ and charge $q_{i}$ ), $V\left(r_{i j}\right)$ is the interaction potential between particles $i$ and $j$, and $\boldsymbol{A}$ is the vector potential.

We start by assuming $\boldsymbol{B}=B(x) \boldsymbol{k}$, and by using the Landau gauge we write the vector potential as $\boldsymbol{A}=A(x) \boldsymbol{j}$, with $\boldsymbol{k}$ and $j$ the versor of the $z$ and $y$ axis, and $A(x)=\int_{x_{0}}^{x} B\left(x^{\prime}\right) d x^{\prime}$, the choice of $x_{0}$ being irrelevant. The equations of motion then read as follows:

$$
\begin{aligned}
\dot{x}_{i} & =\frac{p_{i}^{x}}{m_{i}}, \\
\dot{y}_{i} & =\frac{1}{m_{i}}\left[p_{i}^{y}-q_{i} A\left(x_{i}\right)\right], \\
\dot{z}_{i} & =\frac{p_{i}^{z}}{m_{i}}, \\
\dot{p}_{i}^{x} & =F_{i}^{x}+\frac{q_{i}}{m_{i}}\left[p_{i}^{y}-q_{i} A\left(x_{i}\right)\right] B\left(x_{i}\right), \\
\dot{p}_{i}^{y} & =F_{i}^{y}, \\
\dot{p}_{i}^{z} & =F_{i}^{z},
\end{aligned}
$$

where $F_{i}^{\alpha}=-\frac{\partial \sum_{j \neq i} V\left(r_{i j}\right)}{\partial \alpha_{i}}$ represents the force, deriving from particle-particle interactions, on particle $i$ in direction $\alpha$. Such equations are invariant under the transformation

$$
\mathcal{M}\left(x, y, z, p^{x}, p^{y}, p^{z}, t, \boldsymbol{B}\right) \equiv\left(x,-y, z,-p^{x}, p^{y},-p^{z},-t, \boldsymbol{B}\right) .
$$

Symmetry (3) requires the reversal of time but not of the magnetic field, and therefore standard arguments $[2,17]$ imply the Onsager reciprocal relations. Similar considerations show that Onsager relations remain valid when the magnetic field $\boldsymbol{B}$ varies along any direction in the $x y$ plane. It is important to remark that $V\left(r_{i j}\right)$ is invariant under transformation (3) because we assume that the potential depends only on the distance between particles.

The above reasoning does not apply for a generic magnetic field. Indeed, if we consider $\boldsymbol{B}=B(x, y) \boldsymbol{k}$, and we choose the vector potential as $\boldsymbol{A}=A(x, y) \boldsymbol{j}$, where $A(x, y)=\int_{x_{0}}^{x} B\left(x^{\prime}, y\right) d x^{\prime}$, we can see that transformation (3) implies $p_{i}^{y}-q A\left(x_{i}, y_{i}\right) \rightarrow p_{i}^{y}-q A\left(x_{i},-y_{i}\right)$ and in general $A\left(x_{i},-y_{i}\right) \neq A\left(x_{i}, y_{i}\right)$. To investigate the general case, we shall therefore first turn to numerical simulations and then to physical considerations.

Numerics. We first consider a two-dimensional (2D) gas of interacting particles, of equal mass $m$ and charge $q$ (we set $m=q=1)$. The particles are in a rectangular box of length $L$ (along the $x$ coordinate) and width $W$ (along the $y$ coordinate) (see Fig. 1 for a schematic plot). The system is subject to a magnetic field $B(x, y)$ directed along the $z$ axis. The dynamics is described by the multiparticle collision (MPC) method [22]. The MPC simplifies the numerical simulation of interacting

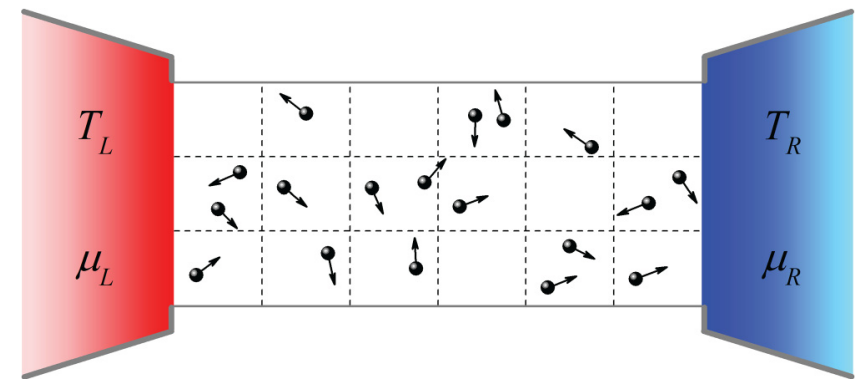

FIG. 1. Schematic drawing of the 2D gas of interacting particles, described by the multiparticle collision dynamics. The cells of dashed-line boundaries represent the partition of space used to model collisions [21]. A magnetic field, transverse to the plane of motion, is applied to the system which is coupled to the left and right electrochemical reservoirs.

particles by coarse-graining the time and space at which interactions occur [21].

The system is placed in contact with two electrochemical reservoirs at $x=0$ and $x=L$, through openings of the same size as the width $W$ of the box. The left and right reservoirs are modeled as ideal gases and are characterized by temperature $T_{\gamma}$ and electrochemical potential $\mu_{\gamma}(\gamma=L, R)$. We use a stochastic model of the reservoirs [23,24]: whenever a particle of the system crosses the boundary which separates the system from the left or right reservoir, it is removed. On the other hand, particles are injected into the system from the boundaries, with rates and energy distribution determined by temperature and electrochemical potential (see, e.g., Ref. [6]). Coupled transport was discussed with this method [25-29], also for the MPC model [30,31].

In our simulations, we set $T_{\gamma}$ and $\mu_{\gamma}$ to be slightly biased from the nominal temperature and electrochemical potential of the system, denoted as $T$ and $\mu$, respectively, to measure the resultant charge and heat current for evaluating the Peltier and Seebeck coefficient [30,31]; i.e., $T_{L, R}=T \pm \Delta T / 2$ and $\mu_{L, R}=\mu \pm \Delta \mu / 2$. In classical physics $\mu$ contains an arbitrary addable constant, and therefore, in addition to $T$ and $\mu$, the particle density $\rho$ must be specified as well.

First, we consider the case $B(x)=g x$. As expected from the above theory, the numerical results of Fig. 2(a) show that the Onsager symmetry $\Pi(g)=T S(g)$ is fulfilled for any value of $g$. In the inset, we show the relative error $\epsilon_{r} \equiv \mid \Pi(g)-$ $T S(g) \mid / \Pi(g)$ for $g=0.3$. We can see that $\epsilon_{r}$, due to the finite integration time $t$ in numerical simulations, decreases $\propto 1 / \sqrt{t}$, as expected for statistical errors, and is smaller than $0.3 \%$ for $t=1.2 \times 10^{8}$.

We then consider the generic case for $2 \mathrm{D}$ systems, and numerically investigate several functions $B(x, y)$, without finding any statistically significant violation of the Onsager symmetry. As an illustrative example, in Fig. 2(b) we show results for $B(x, y)=g \sin [\pi x /(2 L)] \sin [\pi y /(2 W)]$. Similarly to the case of Fig. 2(a), the Onsager symmetry is fulfilled, with the relative error $\epsilon_{r} \propto 1 / \sqrt{t}$ (see the inset, where we show as an example $g=3$, for which $\epsilon_{r}$ is smaller than $0.5 \%$ for $t=1.4 \times 10^{7}$ ).

In fact, our extensive numerical investigations also show that the Onsager symmetry keeps as well in a 

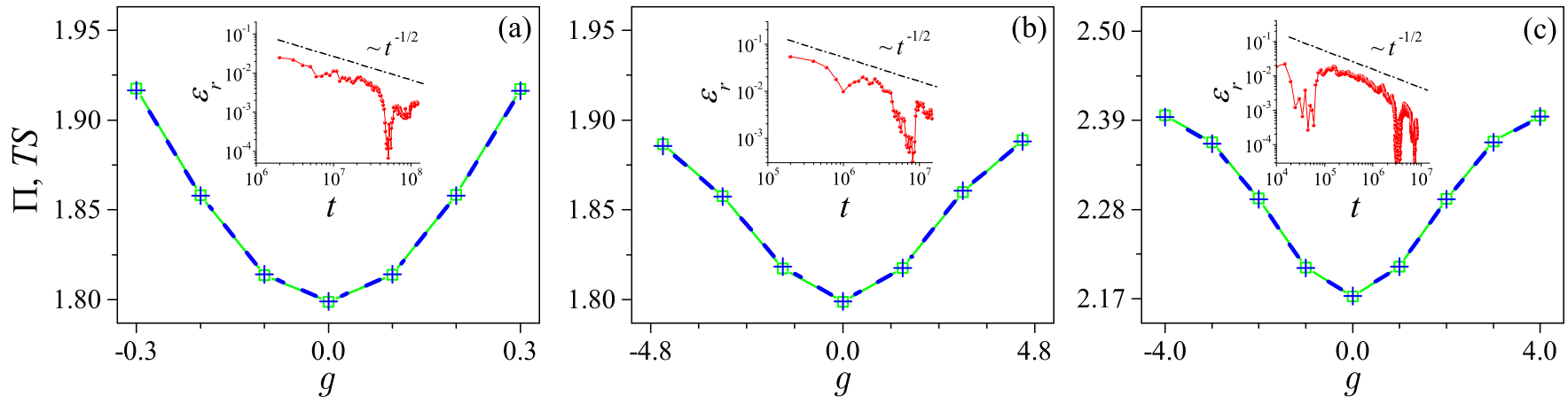

FIG. 2. (a) Peltier coefficient $\Pi$ (green open squares) and thermopower $S$ times temperature $T$ (blue pluses) as a function of $g$ for the 2D interacting gas model subjected to the magnetic field $B(x)=g x$. Parameters for the MPC simulations: length $L=10$, width $W=2$, side of the square cells $a=0.1$, time between collisions $\tau=0.25$, and rotation angle (representing the interaction strength [21]) $\alpha=\pi / 2$. For the reservoirs, the nominal temperature and electrochemical potential of the system is $T=1$ and $\mu=0$, and the corresponding particle density $\rho=$ 22; the temperature (electrochemical potential) bias is $\Delta T=0.05(\Delta \mu=0.05)$. The inset shows the relative error $\varepsilon_{r}=|\Pi(g)-T S(g)| / \Pi(g)$ versus integration time $t$ for $g=0.3$ as an example. (b) Same as (a) but for the magnetic field $B(x, y)=g \sin [\pi x /(2 L)] \sin [\pi y /(2 W)]$. The inset is for the relative error for $g=3$. (c) The corresponding results for the 3D interacting gas model. Parameter values for the MPC simulations are $L=10, W=H=2, a=0.1$, and $\tau=0.25$. For the reservoirs, $T=1, \mu=0$, and the corresponding particle density $\rho=88$; the temperature (electrochemical potential) bias of the reservoirs is $\Delta T=0.05(\Delta \mu=0.05)$. Inset: relative error $\varepsilon_{r}$ for $g=3$.

three-dimensional (3D) system subjected to an arbitrary magnetic field with varying amplitude and direction in space. As an example, we present in Fig. 2(c) the results for a 3D extension of the 2D gas model discussed previously. In this model, all gas particles (of unit mass and charge) are confined to move in a cuboid-shaped volume of length $L$, width $W$, and height $H$ in the $x, y$, and $z$ coordinate, respectively. The MPC dynamics is assumed again [21]. At the two ends in the longitudinal direction, the system exchanges particles and energy with two reservoirs modeled by 3D ideal gases of temperatures $T_{L, R}=$ $T \pm \Delta T / 2$ and electrochemical potentials $\mu_{L, R}=\mu \pm \Delta \mu / 2$, through two openings of area $W H$, in a similar way as in the $2 \mathrm{D}$ case. For all magnetic fields that have been simulated with, we find that the Onsager symmetry holds to a satisfactory accuracy. For the data shown in Fig. 2(c) as an example, the particular magnetic field is $\boldsymbol{B}=g\left(B_{x}, B_{y}, B_{z}\right)$, with $B_{x}=f_{y} f_{z}$, $B_{y}=f_{z} f_{x}$, and $B_{z}=f_{x} f_{y}$, where $f_{x}=\sin [\pi x /(2 L)], f_{y}=$ $\sin [\pi y /(2 W)]$, and $f_{z}=\sin [\pi z /(2 H)]$.

Discussion and conclusions. The results for 2D systems can be understood from the following argument [32]. A generic field $B(x, y)$ can be approximated by a finite number $n$ of step functions (in the $y$ direction), $B(x, y) \approx B\left(x, y_{k}\right)$ for step $k(k=1, \ldots, n)$. For each step the magnetic field is constant in the $y$ direction, and therefore symmetry (3) applies. On the other hand, discontinuities of the field between steps would induce sudden changes of velocity but not affect the symmetry. A similar argument holds for 3D motion. Here, one could divide the system into small volumes $d V_{\alpha}$, and for each volume approximate the magnetic field with a constant vector. Building a local Cartesian tern $\left(x_{\alpha}, y_{\alpha}, z_{\alpha}\right)$ for each $d V_{\alpha}$, with $z_{\alpha}$ pointing in the field direction, symmetry (3) applies locally. For $d V_{\alpha} \rightarrow 0$, we thus expect to reverse each trajectory by locally applying symmetry (3), without reversing the magnetic field.

The results of this Rapid Communication could be extended to quantum mechanics, with the proper counterpart of map $\mathcal{M}$ of Eq. (3) discussed in Ref. [19]. We expect that Onsager relations remain valid under other time-reversal breaking mechanisms like the Coriolis force, even though the question remains open.

Onsager reciprocity relations are linear response relations [33] and therefore cannot claim the universal validity of the first and second law of thermodynamics. However, the results presented here show that Onsager relations can hold for systems without time-reversal symmetry, significantly extending the applicability of one of the most powerful principles of nonequilibrium thermodynamics.

Acknowledgments. We thank Keiji Saito for bringing to our attention Ref. [17] and Lamberto Rondoni for useful discussions. We acknowledge support by the National Natural Science Foundation of China (Grants No. 11535011 and No. 11335006) and by the Istituto Nazionale di Fisica Nucleare through the project QUANTUM.
[1] L. Onsager, Phys. Rev. 37, 405 (1931).

[2] H. B. Callen, Thermodynamics and an Introduction to Thermostatics, 2nd ed. (Wiley, New York, 1985).

[3] S. R. de Groot and P. Mazur, Nonequilibrium Thermodynamics (North-Holland, Amsterdam, 1962).

[4] H. B. G. Casimir, Rev. Mod. Phys. 17, 343 (1945).
[5] G. Benenti, K. Saito, and G. Casati, Phys. Rev. Lett. 106, 230602 (2011).

[6] G. Benenti, G. Casati, K. Saito, and R. S. Whitney, Phys. Rep. 694, 1 (2017).

[7] K. Saito, G. Benenti, G. Casati, and T. Prosen, Phys. Rev. B 84, 201306(R) (2011).

[8] D. Sánchez and L. Serra, Phys. Rev. B 84, 201307(R) (2011). 
[9] M. Horvat, T. Prosen, G. Benenti, and G. Casati, Phys. Rev. E 86, 052102 (2012).

[10] V. Balachandran, G. Benenti, and G. Casati, Phys. Rev. B 87, 165419 (2013).

[11] K. Brandner, K. Saito, and U. Seifert, Phys. Rev. Lett. 110, 070603 (2013).

[12] K. Brandner and U. Seifert, New J. Phys. 15, 105003 (2013).

[13] K. Brandner and U. Seifert, Phys. Rev. E 91, 012121 (2015).

[14] K. Yamamoto, O. Entin-Wohlman, A. Aharony, and N. Hatano, Phys. Rev. B 94, 121402(R) (2016).

[15] N. Shiraishi, K. Saito, and H. Tasaki, Phys. Rev. Lett. 117, 190601 (2016).

[16] S. Datta, Electronic Transport in Mesoscopic Systems (Cambridge University Press, Cambridge, England, 1995).

[17] S. Bonella, G. Ciccotti, and L. Rondoni, Europhys. Lett. 108, 60004 (2014).

[18] S. Bonella, A. Coretti, L. Rondoni, and G. Ciccotti, Phys. Rev. E 96, 012160 (2017).

[19] P. De Gregorio, S. Bonella, and L. Rondoni, Symmetry 9, 120 (2017).

[20] A. Coretti, S. Bonella, L. Rondoni, and G. Ciccotti, Mol. Phys. 116, 3097 (2018)

[21] See Supplemental Material at http://link.aps.org/supplemental/ 10.1103/PhysRevResearch.2.022009 for details on numerical simulations.
[22] A. Malevanets and R. Kapral, J. Chem. Phys. 110, 8605 (1999).

[23] C. Mejía-Monasterio, H. Larralde, and F. Leyvraz, Phys. Rev. Lett. 86, 5417 (2001).

[24] H. Larralde, F. Leyvraz, and C. Mejía-Monasterio, J. Stat. Phys. 113, 197 (2003).

[25] G. Casati, C. Mejía-Monasterio, and T. Prosen, Phys. Rev. Lett. 101, 016601 (2008).

[26] K. Saito, G. Benenti, and G. Casati, Chem. Phys. 375, 508 (2010).

[27] G. Benenti, G. Casati, and J. Wang, Phys. Rev. Lett. 110, 070604 (2013).

[28] J. Stark, K. Brandner, K. Saito, and U. Seifert, Phys. Rev. Lett. 112, 140601 (2014).

[29] S. Chen, J. Wang, G. Casati, and G. Benenti, Phys. Rev. E 92 , 032139 (2015).

[30] G. Benenti, G. Casati, and C. Mejía-Monasterio, New J. Phys. 16, 015014 (2014).

[31] R. Luo, G. Benenti, G. Casati, and J. Wang, Phys. Rev. Lett. 121, 080602 (2018).

[32] This argument develops a suggestion by L. Rondoni (private communication).

[33] However, for a recent extension of the Onsager reciprocal relation to far from equilibrium systems, see H. Vroylandt, D. Lacoste, and G. Verley, J. Stat. Mech. (2019) 054002. 